\title{
Interactive Holographic Pyramid Using Two-Layer Integral Photography
}

\author{
Toshiaki Yamanouchi ${ }^{1}$, Shohei Anraku${ }^{1}$, Nahomi Maki $^{2}$, Kazuhisa Yanaka ${ }^{1}$ \\ ${ }^{1}$ Kanagawa Institute of Technology \\ 1030 Shimo-ogino, Atsugi-shi, Kanagawa-ken, 243-0292 Japan \\ yama@ic.kanagawa-it.ac.jp; s1785004@cce.kanagawa-it.ac.jp, yanaka@ic.kanagawa-it.ac.jp \\ ${ }^{2}$ Tokyo University of the Arts (Bankokubashi Campus) \\ 4-32 Kaigan-dori, Naka-ku, Yokohama-shi, Kanagawa-ken, 231-0002 Japan \\ maki.nahomi@fm.geidai.ac.jp
}

\begin{abstract}
We propose a new 3D holographic pyramid system that can represent a wide range of depth by using two integral photography (IP) displays, each of which consists of a liquid crystal display and a fly's eye lens. The IP displays are placed on the top and bottom of the pyramid, respectively. We also introduce interactivity in which the displayed object moves by detecting movement of a user's hand by using Leap Motion to enhance the stereoscopic effect.
\end{abstract}

Keywords: Holographic Pyramid, Two-Layer Integral Photography, Fly’s Eye Lens, Leap Motion.

\section{Introduction}

The holographic pyramid, which consists of a liquid crystal display (LCD) and a quadrangular pyramid typically made of a transparent plastic material, is a popular way to make an image appear floating around the center of the pyramid when it is observed from the surroundings. In fact, the image displayed on the LCD is reflected on the outer surface of the pyramid.

Meanwhile, integral photography (IP) [1] is an excellent 3D image display system because it provides not only horizontal but also vertical parallax without the need for wearing stereo glasses. Specifically, when a normal LCD of a holographic pyramid is replaced with an IP display, which consists of a fly's eye lens and an LCD, the floating image also becomes 3D [2]. Figure 1 shows the structure of a conventional holographic pyramid using IP. The image of the LCD located at the lower right of the pyramid appears as a 3D image when viewed through the fly's eye lens. Given that this image is directly reflected on the outer surface of the pyramid, a 3D image appears to float inside the pyramid when viewed from the right side. However, the 3D image looks blurred when it is located far away in front or back from the center of the pyramid because the density of light ray decreases.

\section{Method}

To solve the aforementioned problem, we propose a novel display system in which the top IP display is placed upside down on the pyramid as shown in Figure 2. The image of the top IP display located on the upper left of the pyramid forms a 3D image via the fly's eye lens. This 3D image is reflected on the inner surface of the pyramid. Thus, a 3D image appears to float outside the pyramid when viewed from the right side. As a result, 3D images created by the bottom and top IP displays can be exhibited at physically distant places inside and outside the pyramid. Notably, the reflection on the inner surface of the pyramid has not been used in conventional holographic pyramid. The proposed display system is a two-layer IP display.

Leap Motion is also used to introduce interactivity and thus enhance the stereoscopic effect. 


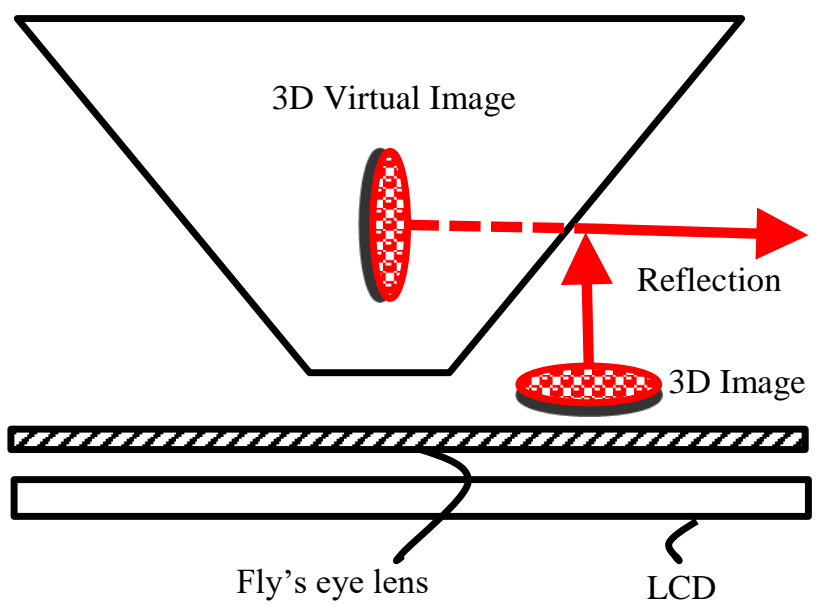

Fig. 1: Holographic pyramid system using IP.

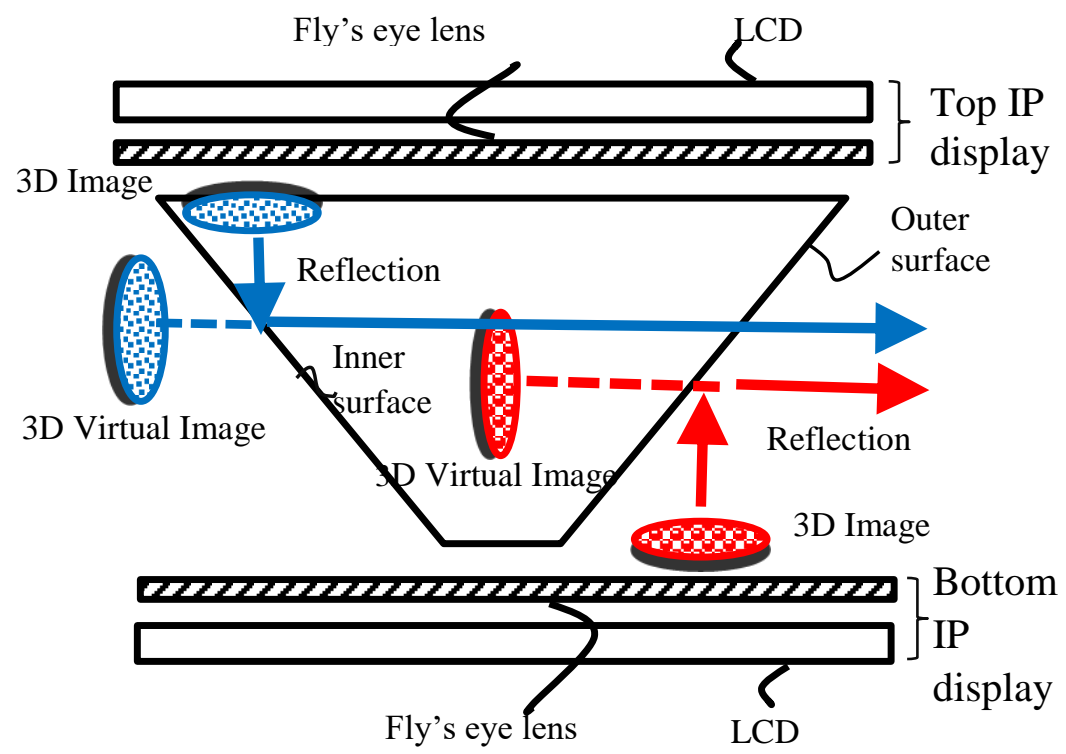

Fig. 2: New holographic pyramid system using two-layer IP.

\section{Experiment}

Figure 3 shows the IP image of a 3DCG goldfish displayed on the bottom LCD. Given that the goldfish has animation and interaction, this image should be rendered in real time. We use Unity because it is suitable for the purpose [3-4].

Figure 4 shows the image of 3DCG water plants behind the goldfish displayed on the top LCD.

Figure 5 shows the outlook of our experimental system. When observing from four sides of the pyramid, the goldfish seen from four different directions appears swimming in the pyramid. The background water plants are clear despite the fact that they are displayed fairly deeper than the goldfish. 


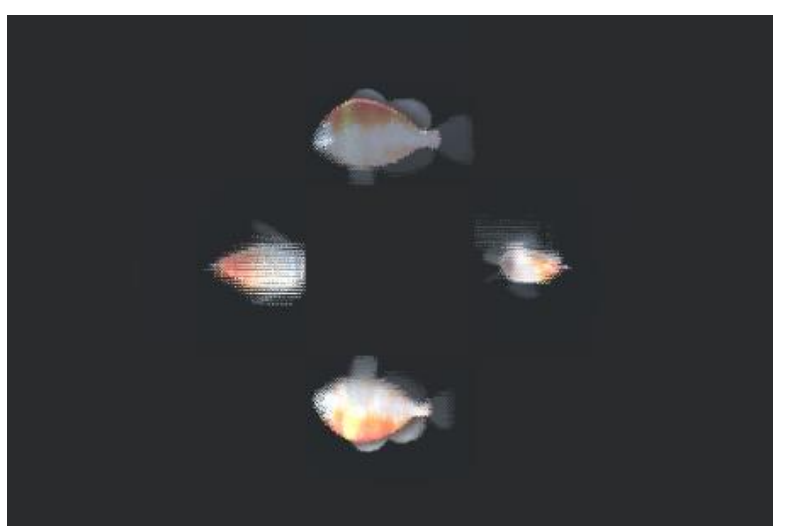

Fig. 3: Image displayed on the bottom LCD.

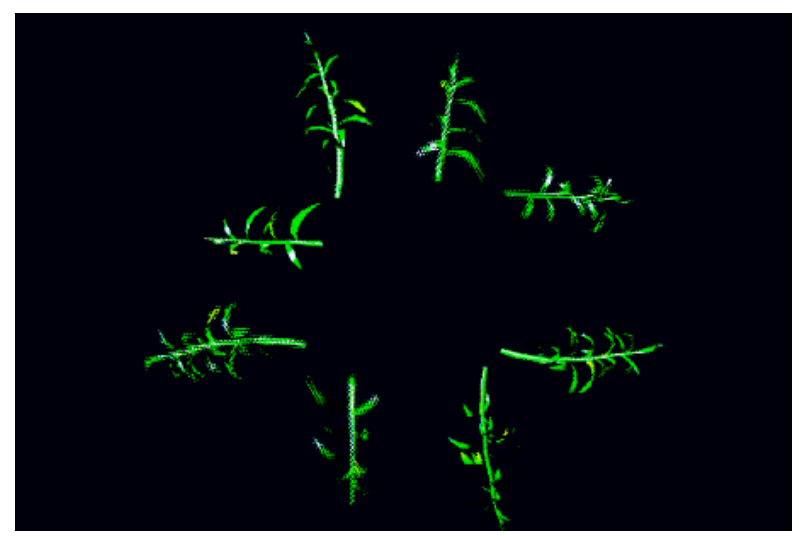

Fig. 4: Image displayed on the top LCD.

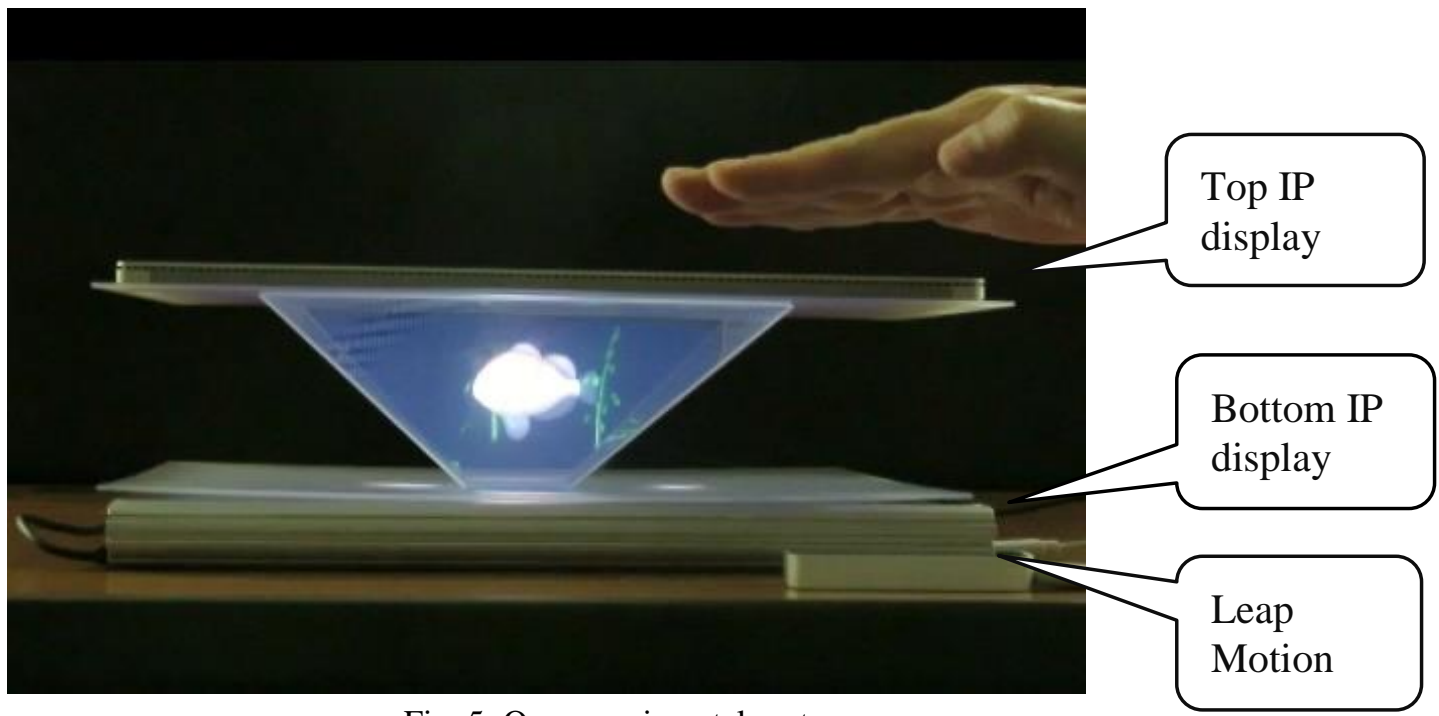

Fig. 5: Our experimental system. 


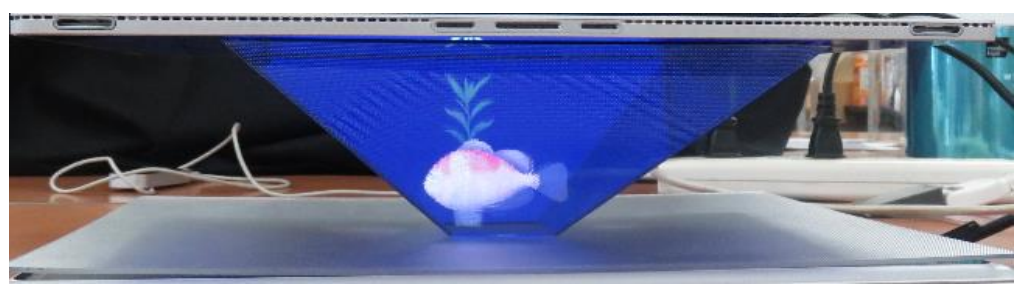

(a) Front side

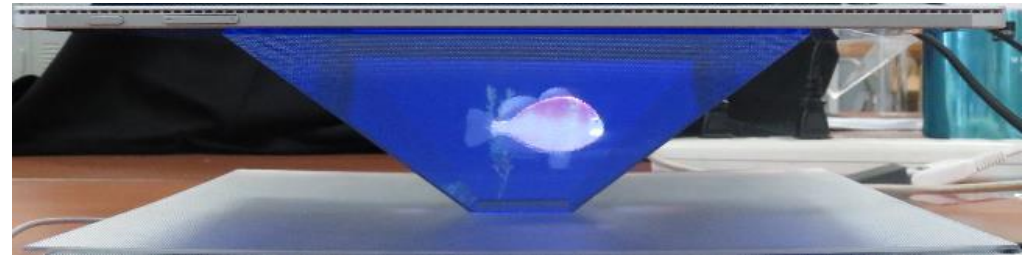

(b) Back side

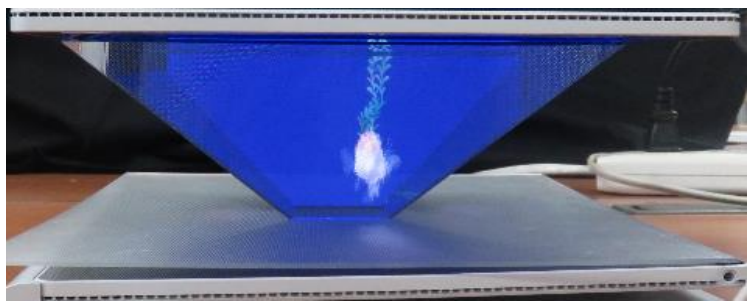

(c) Left side

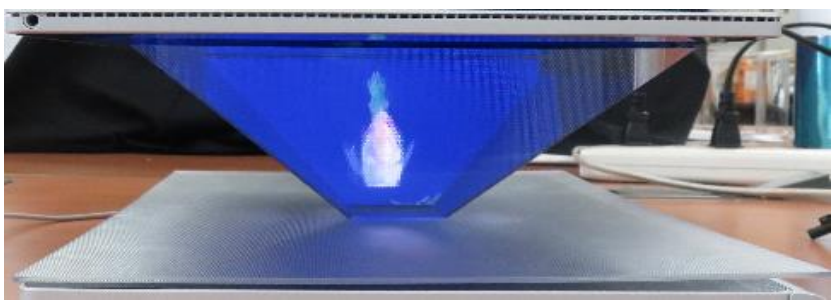

(d) Right side

Fig. 6: Pictures of the pyramid viewed from four directions.

We use two laptop computers, each of which is a Microsoft Surface Book with an LCD size of 13.5 inches and an LCD pixel number of 3,000 $\times 2,000$. A hexagonal fly's eye lens with a lens pitch of $1 \mathrm{~mm}$ is attached to each LCD.

A Leap Motion, which is a motion capture device for human hands and fingers, is attached to the lower laptop PC. If the Leap Motion detects movement from at least one hand, the goldfish moves upward slowly; otherwise, the goldfish moves downward slowly. Such a simple interaction is effective for enhancing the sense of depth.

\section{Conclusions}

We develop a novel two-layer IP system by extending existing holographic pyramid system using IP. In our system, the top IP display is placed upside down on the pyramid in addition to the bottom IP display placed below the pyramid. In this way, the optical reflections on the inner and outer sides of the pyramid are effectively utilized, and two virtual images can be produced at mutually separate places. When the goldfish is displayed on the bottom IP display and the water plants behind it are displayed on the top IP display, the parallax becomes large and strong sense of depth can be obtained. This display can be observed from any direction because it has a square pyramid shape. Four different 3D images can also be displayed at the same time. The system can be installed in public places and can be used for various purposes, such as public signage.

\section{Acknowledgements}

This work was supported by KAKENHI Grant Number 16K00284.

\section{References}

[1] M. G. Lippmann, "Epreuves, reversibles donnant la sensation du relief," in J. Phys., vol. 4, pp. 821-825, 1908.

[2] T. Yamanouchi, N. Maki and K. Yanaka, "Holographic Pyramid Using Integral Photography," in Proceedings of EECSS'16, Paper No. MHCI 109, pp. 1-4. 2016. 
[3] S. Anraku, F. Ishiwata, N. Maki, T. Yamanouchi and K. Yanaka, "Real-Time Integral Photography Using a Game Engine," in SIGGRAPH 2017 Posters, 2017.

[4] S. Anraku, T. Yamanouchi and K. Yanaka, "Real-Time Integral Photography Holographic Pyramid Using a Game Engine,” in VISIGRAPP 2018 Proceedings, vol. 4, VISAPP, pp. 603-607, 2018. 